\title{
Actualización de la resistencia a antimicrobianos de bacilos Gram negativos aislados en hospitales de nivel III de Colombia: años 2006, 2007 y 2008
}

David Felipe Briceño, Adriana Correa, Carlos Valencia, Julián Andrés Torres, Robinson Pacheco, María Camila Montealegre, Diego Ospina, María Virginia Villegas y Grupo de Resistencia

Bacteriana Nosocomial de Colombia

Centro Internacional de Entrenamiento e Investigaciones Médicas, CIDEIM, Cali, Colombia

El Grupo para el Estudio de la Resistencia Nosocomial en Colombia está conformado por las siguientes ciudades, hospitales y personas.

Bogotá: Hospital Central de la Policía: Henry Mendoza, Flor Ángela Cubides, Martha Patricia Meléndez, Luz Angélica Quintero, Alba Lucía Sanín, Nancy Botia, Sandra Reina; Hospital Santa Clara: Beatriz E. Porras, Guillermo Ortiz, Luz Mila López; Hospital Militar Central: Henry Oliveros, María Nilse González, Ángela Pescador, Mónica Ballesteros, Sandra Valderrama, Alirio Rodríguez, Héctor Posso, Gloria Gallo, Fernando Guzmán, Jairo Pérez. Medellín: Hospital Pablo Tobón Uribe: Carlos Ignacio Gómez, Jaime López, Jorge Donado, Mónica Cuartas, Ana Lucía Correa, Lina Marcela Castañeda; Clínica Las Américas: Julián Betancourth, Juan David Villa, Jorge Nagles, Ana Cristina Quiroga, Nancy Estella González, Magda Orjuela, Rodrigo Agudelo; Hospital General de Medellín: Marta Vallejo, Luz Marina Melguizo, Victoria García, Dora Rivas, Rubén Darío Trejos, Alberto Pérez, Sergio A. Vélez.

Cali: Hospital Universitario del Valle: Ernesto Martínez, José Millán Oñate, Lena Barrera, Cristian Payares, Luz Marina Gallardo, Alba Lucía Bohórquez, Nancy Villamarín; Fundación Clínica Valle del Lili: Fernando Rosso, Alejandra Toala, John Jairo Echeverri, Mónica Recalde, Juan Diego Vélez, José García.

Bucaramanga: Fundación Cardiovascular: Beatriz E. López, Claudia Bárcenas, Adriana Pinto; Clínica La Foscal: Karol Monsalve, Luis Ángel Villar, Mayerly Anaya Uribe; Hospital Universitario de Santander: Agustín Vega, Myriam Fanny Amaya, Martha Jácome, Rocío Abaúnza, Martín Mejía.

Barranquilla: Clínica General del Norte: Iván Zuluaga, Rubén Camargo, Adriana Marín, Ángela Mendoza.

Pereira: Hospital Universitario San Jorge: Carmen Elisa Llano, Myriam Gómez, Rodolfo Cabrales, Araceli Cano, Juan Cobo, Martha Lucía Gómez.

Ibagué: Hospital Federico Lleras Acosta: Claudia Echeverri, Amparo Ovalle, María del Rosario Aldana, Pablo López.

Introducción. La resistencia a antimicrobianos se ha identificado como uno de los mayores problemas de salud pública, por lo cual debe ser vigilada permanentemente a través de sistemas efectivos de vigilancia.

Objetivo. Describir el comportamiento de la resistencia antimicrobiana de bacilos Gram negativos, en las instituciones hospitalarias pertenecientes al Grupo para el Estudio de la Resistencia Nosocomial en Colombia, de enero 2006 a diciembre 2008.

Materiales y métodos. Es un estudio descriptivo realizado con la información de los perfiles de sensibilidad bacteriana suministrada por 14 hospitales de tercer nivel en siete ciudades de Colombia, definidos como el Grupo para el Estudio de la Resistencia Nosocomial en Colombia, de enero de 2006 a diciembre de 2008.

Usando el software WHONET 5.4, se describe el comportamiento de la resistencia bacteriana de las especies de Enterobacteriaceae (Escherichia coli, Klebsiella pneumoniae y Enterobacter cloacae) y bacilos Gram negativos no fermentadores (Pseudomonas aeruginosa y Acinetobacter baumannii) frente a antibióticos seleccionados como marcadores de resistencia en salas generales y unidades de cuidados intensivos de pacientes adultos.

Resultados. En algunas Enterobacteriaceae se observó disminución de la frecuencia de resistencia antimicrobiana, mientras que $P$. aeruginosa se consolida como un germen multirresistente, cuyas frecuencias de resistencia continúan en aumento.

Conclusiones. Los programas de vigilancia pueden tener un impacto en la disminución significativa de gérmenes multirresistentes, ya que contribuyen a la adecuada implementación de protocolos encaminados a fortalecer las estrategias de control de infecciones y el manejo racional de antibióticos en cada hospital.

Palabras clave: bacterias Gram negativas, farmacorresistencia bacteriana, vigilancia epidemiológica, Colombia 


\section{Antimicrobial resistance of Gram negative bacilli isolated from terciary-care hospitals in Colombia}

Introduction. Antimicrobial resistance has been identified as one of the major public health problems worldwide. To facilitate its control, bacterial resistance levels must be monitored permanently by effective surveillance systems.

Objective. To describe the antimicrobial resistance patterns of Gram negative bacilli in Colombian hospitals over a 3-year period.

Materials and methods. This descriptive study used the bacterial susceptibility profiles provided by 14 tertiary-care hospitals belonging to the Colombian Nosocomial Resistance Study Group. The hospitals were located in 7 major cities in Colombia, and provided records over the period January 2006 to December 2008. Using WHONET 5.4, the antimicrobial resistance patterns were described for the Enterobacteriaceae (Escherichia coli, Klebsiella pneumoniae and Enterobacter cloacae) and non-fermenters (Pseudomonas aeruginosa and Acinetobacter baumannii). Comparisons were made in the bacterial responses to selected antibiotics in samples from general wards and from adult intensive care units.

Results. The antimicrobial resistance frequencies of several Enterobacteriaceae species showed a decreasing trend. In contrast, $P$. aeruginosa was demonstrated to be a multidrug-resistance organism with increasing resistance frequencies.

Conclusions. These data emphasize the importance of surveillance programs in detecting presence of multidrug-resistant organisms. This information will aid the implementation of protocols aimed to strengthen the infection control strategies and antibiotic stewardship in each hospital.

Key words: Gram-negative bacteria; drug resistance, bacterial; epidemiologic surveillance, Colombia

La resistencia a antimicrobianos se ha identificado como uno de los mayores problemas de salud pública a nivel mundial (1), por lo cual requiere un seguimiento permanente a través de sistemas efectivos de vigilancia (2). Esto se ha documentado desde 1970, en un estudio sobre la eficacia del control de infecciones hospitalarias (Study on the Efficacy of Nosocomial Infection Control, SENIC) en Estados Unidos, al mostrar que la vigilancia intensiva, junto con otros programas de control de infecciones, se asociaba con una reducción significativa de las tasas de este tipo de infecciones (3).

Actualmente, varias organizaciones oficiales en el mundo se dedican a la vigilancia activa de la resistencia antimicrobiana. Entre ellas se encuentran el European Antimicrobial Resistance Surveillance System(EARSS) y, en Estados Unidos, la National Nosocomial Infections Surveillance (NNIS), la Intensive Care Antimicrobial Resistance Epidemiology (ICARE) y el National Antimicrobial Resistance Monitoring System (NARMS), entre otros.

Por otro lado, entre los programas de vigilancia global más reconocidos que operan actualmente

\footnotetext{
Correspondencia:

Maria Virginia Villegas, Centro Internacional de Entrenamiento e Investigaciones Médicas (CIDEIM), Carrera 125 № 19-225, Cali, Colombia

Teléfono: (+572) 555 2164; fax: (+572) 5552638.

mariavirginia.villegas@gmail.com

Recibido: 29/08/09; aceptado:13/04/10
}

están: el SENTRY Antimicrobial Surveillance Program, creado en 1997, mide la frecuencia de patógenos y los patrones de resistencia antimicrobiana en infecciones hospitalarias y adquiridas en la comunidad; el Meropenem Yearly Susceptibility Test Information Collection (MYSTIC), creado en 1997 como un estudio longitudinal que brinda información de la sensibilidad a meropenem de los agentes patógenos hospitalarios, y el proyecto Alexander, creado en 1992, el cual hace seguimiento a los gérmenes causantes de infecciones de las vías respiratorias inferiores adquiridas en la comunidad, con el fin de resaltar el número limitado de antimicrobianos orales (4).

Según la Organización Mundial de la Salud (OMS) (1), entre los factores considerados como causantes de la resistencia antimicrobiana, están el uso inapropiado de antibióticos, la falta de sistemas de vigilancia efectivos en cada país y región, la ausencia de legislación que permita el control en el mercado de la venta de medicamentos en las farmacias y el uso extendido de antibióticos en animales destinados para el consumo humano.

La identificación y vigilancia de nuevos patrones de resistencia a nivel local, nacional y global, permiten un análisis de la distribución y comportamiento de los patógenos multirresistentes; ésta se define como resistencia a tres o más clases de antibióticos activos contra un germen (5) que, a su vez, permite el diseño y la implementación de guías sobre el uso racional de antibióticos (6), lo cual 
evita la instauración de terapias antimicrobianas inapropiadas y ayuda a disminuir la mortalidad hospitalaria (7). Además, el análisis de los datos por medio de los sistemas de vigilancia permite evaluar la efectividad de las intervenciones establecidas para contener la resistencia (6).

En Colombia, la magnitud del problema ha fomentado la implementación de sistemas de vigilancia por diversos grupos (8). El Centro Internacional de Entrenamiento e Investigaciones Médicas, CIDEIM, ha estudiado y reportado desde el año 2001 diferentes mecanismos y patrones de resistencia en bacterias Gram negativas hospitalarias (9-11); para ello, ha constituido una red de 14 hospitales de tercer nivel en siete ciudades principales del país, al que ha denominado Grupo para el Estudio de la Resistencia Nosocomial en Colombia. Esta información ha servido como base para trabajar con los comités de infecciones hospitalarias, buscando herramientas para entender y disminuir el problema de la resistencia a los antibióticos. Nuestro grupo publicó en el año 2006 los resultados de esta red entre los años 2003 y 2005, y se propuso la necesidad de crear una red nacional de vigilancia de la resistencia a antimicrobianos para evaluar el comportamiento local y nacional de los patrones de resistencia (12).

En esta comunicación se presenta una descripción de la resistencia antimicrobiana de bacilos Gram negativos en los hospitales de Colombia pertenecientes este grupo, durante los años 2006 a 2008.

\section{Materiales y métodos}

\section{Diseño del estudio}

Es un estudio descriptivo realizado con la información de los perfiles de sensibilidad bacteriana suministrada por 14 hospitales de tercer nivel en siete ciudades pertenecientes al Grupo para el Estudio de la Resistencia Nosocomial en Colombia, durante el periodo comprendido entre el $1^{\circ}$ de enero de 2006 y el 31 de diciembre de 2008.

Se describen la frecuencia y los perfiles de sensibilidad de las especies de Enterobacteriaceae (Escherichia coli, Klebsiella pneumoniae y Enterobacter cloacae) y bacilos Gram negativos no fermentadores (Pseudomonas aeruginosa y Acinetobacter baumannii) frente a antibióticos seleccionados como marcadores de resistencia: piperacilina/tazobactam, ceftriaxona, cefotaxime, ceftazidime, cefepime, amicacina, ciprofloxacina, imipenem y meropenem, tamizados en microorganismos aislados de pacientes hospitalizados en salas generales y unidades de cuidados intensivos de adultos.

\section{Población de estudio}

Se contó con la participación de 14 hospitales de tercer nivel de atención en salud en siete ciudades principales de Colombia. Las instituciones participantes fueron: el Hospital Central de la Policía, el Hospital Militar Central y el Hospital Santa Clara, en Bogotá; la Fundación Clínica Valle del Lili y el Hospital Universitario del Valle, en Cali; la Clínica Las Américas, el Hospital General de Medellín y el Hospital Pablo Tobón Uribe, en Medellín; la Clínica General del Norte, en Barranquilla; la Clínica La Foscal, la Fundación Cardiovascular y el Hospital Universitario de Santander, en Bucaramanga; el Hospital Universitario San Jorge, en Pereira, y el Hospital Federico Lleras Acosta, en Ibagué.

Estas instituciones cuentan con un promedio de 320 camas (intervalo, 160-725) en salas generales y 48 camas (intervalo, 12-125) en unidades de cuidados intensivos de adultos; ocho de ellos son públicos, cuatro privados y dos de régimen especial; siete cuentan con programa de trasplantes.

Todos los hospitales aplican la definición de infección hospitalaria de los Centers for Disease Control and Prevention (CDC) de Atlanta, Estados Unidos (13).

Los antibióticos tamizados tienen actividad reconocida contra bacilos Gram negativos de acuerdo con las guías del Clinical and Laboratory Standards Institute (CLSI) (14). Se escogieron estos antibióticos $\beta$-lactámicos por ser marcadores para la detección de $\beta$-lactamasas de espectro extendido (BLEE), AmpC y carbapenemasas. En relación con la resistencia a las quinolonas, se seleccionó la ciprofloxacina como marcador de esta familia contra bacterias Gram negativas; para la resistencia a los aminoglucósidos, se escogió la amicacina por su mayor estabilidad frente a las enzimas modificadoras de aminoglucósidos producidas por estas bacterias (14).

Para realizar las pruebas de sensibilidad bacteriana a los antibióticos, 13 hospitales usaron sistemas automatizados (2, Microscan; 7, Vitek 2; 1, Vitek 1; 1 , Autoscan 4; y 2, Phoenix) y uno usó un método manual (difusión de disco Kirby-Bauer).

Todos los laboratorios tienen control de calidad interno y externo. El control interno lo realizan 
con cepas del ATCC (Escherichia coli ATCC ${ }^{\circledR}$ 25922, Klebsiella pneumoniae ATCC® 700603, Pseudomonas aeruginosa ATCC ${ }^{\circledR}$ 27853, Acinetobacter baumannii ATCC $\circledast$ 17978) y el externo, a través de distintas instituciones, incluyendo: Instituto Nacional de Salud, Secretaría de Salud Municipal y Departamental, PROASECAL, Biomerieux, Medical Laboratory Evaluation (MLE).

\section{Recolección y manejo de la información}

EI CIDEIM estandarizó el proceso de recolección y manejo de la información (12). Mensualmente se recibieron por internet los perfiles de sensibilidad bacteriana de cada hospital, obtenidos directamente de los sistemas automatizados del laboratorio. La información recibida se convirtió mediante el sistema de conversión de datos BacLink, versión 2.0, y se analizó por medio del software de análisis de sensibilidad antimicrobiana de la Organización Mundial de la Salud (WHONET, versión 5.4, OMS 2009) (15). El hospital que usó el método manual envió los perfiles de sensibilidad bacteriana convertidos en formato WHONET.

\section{Análisis de la información}

Se analizaron los aislamientos provenientes de los pacientes hospitalizados en salas generales y unidades de cuidados intensivos de adultos. Sólo se tomó en cuenta el primer aislamiento de cada paciente, con el fin de estandarizar el análisis y evitar la amplificación de la resistencia. Se determinaron los porcentajes de frecuencias y resistencias durante los años 2006, 2007 y 2008, los cuales se compararon, y se determinó su tendencia por medio de la prueba estadística de ji al cuadrado de tendencia lineal, con un nivel de significancia de 0,05 . El software estadístico utilizado para los análisis fue Epi-Info, versión 6.04.

\section{Actividades realizadas en el CIDEIM}

Durante el periodo de estudio, el CIDEIM elaboró reportes semestrales con los resultados del estudio a los comités de infectología y a los laboratorios de microbiología de las instituciones.

Además del análisis de los perfiles de sensibilidad bacteriana, el CIDEIM analizó las cepas enviadas por estos hospitales con perfiles de resistencia definidos previamente, con el fin de realizar pruebas moleculares para la identificación y caracterización de $\beta$-lactamasas (especialmente, carbapenemasas). La descripción de estos procedimientos moleculares y sus hallazgos será motivo de otra publicación.
Estos reportes incluyeron la distribución de las frecuencias de gérmenes en cada sala, la distribución de las concentraciones inhibitorias mínimas (CIM) y los porcentajes de resistencia a antibióticos seleccionados, descritos previamente. Además, se incluyó un análisis que contiene reportes de errores mayores o menores de sensibilidad de las cepas enviadas y los resultados de la prueba para detección de BLEE y carbapenemasas con su correspondiente análisis de reacción en cadena de la polimerasa (PCR), y, además, se realizó electroforesis de campos pulsados (EFCP) para la vigilancia de brotes en grupos seleccionados de bacterias en las unidades de cuidados intensivos.

\section{Recomendaciones del CIDEIM a los hospitales}

De acuerdo con el perfil epidemiológico de cada institución, que incluía el comportamiento de la resistencia bacteriana caracterizada por métodos moleculares y los resultados de la EFCP, el CIDEIM hizo una serie de recomendaciones dirigidas al manejo de las bacterias resistentes, al uso racional de los antibióticos y a medidas de control de infección, como barreras de contacto, lavado de manos y desinfección de equipos y superficies. De igual manera, hizo el acompañamiento a los hospitales dos veces por año para reforzar la información y las recomendaciones hechas en el informe. Sin embargo, es importante mencionar que no se determinó el cumplimiento ni el impacto de estas intervenciones en las instituciones.

\section{Resultados}

\section{Distribución de microorganismos en salas generales y unidades de cuidados intensivos}

Durante el periodo estudiado, en salas generales se reportaron 46.195 aislamientos. El número de gérmenes aislados aumentó significativamente en el transcurso de los tres años, y se encontró que los cinco Gram negativos más frecuentes en el 2008 fueron: E. coli (26\%), K. pneumoniae (9\%), $P$. aeruginosa (7\%), E. cloacae (3\%) y A. baumannii (1\%). De los Gram negativos reportados, hubo incremento significativo en $E$. coli, Proteus mirabilis y $P$. aeruginosa, contrario a lo observado con $A$. baumannii, que mostró una tendencia a la disminución ( $p \leq 0,001)$ (cuadro 1).

En las unidades de cuidados intensivos se reportaron 22.344 aislamientos durante los tres años. De ellos, los cinco Gram negativos más frecuentes en el 2008 fueron: E. coli (15\%), K. pneumoniae (11\%), $P$. aeruginosa (9\%), E. cloacae (3\%) y A. baumannii (3\%). Al igual que en salas 
Cuadro 1. Microorganismos más frecuentes en salas generales de 14 hospitales de nivel III de Colombia, años 2006,2007 y 2008.

\begin{tabular}{|c|c|c|c|c|c|c|c|}
\hline \multirow[t]{3}{*}{ Especie } & \multicolumn{7}{|c|}{ Proporción de aislamientos } \\
\hline & \multicolumn{2}{|c|}{2006} & \multicolumn{2}{|c|}{2007} & \multicolumn{2}{|c|}{2008} & \multirow[t]{2}{*}{$P$} \\
\hline & $\mathbf{n}$ & $\%$ & $\mathbf{n}$ & $\%$ & $\mathbf{n}$ & $\%$ & \\
\hline Escherichia coli & 2.365 & $22 \%$ & 3.957 & $27 \%$ & 5.406 & $26 \%$ & $\leq 0,001$ \\
\hline Staphylococcus aureus & 1.397 & $13 \%$ & 1.905 & $13 \%$ & 2.287 & $11 \%$ & $\leq 0,001$ \\
\hline Klebsiella pneumoniae & 967 & $9 \%$ & 1.319 & $9 \%$ & 1.871 & $9 \%$ & 0,997 \\
\hline Pseudomonas aeruginosa & 860 & $8 \%$ & 1.172 & $8 \%$ & 1.455 & $7 \%$ & $\leq 0,001$ \\
\hline Staphylococcus epidermidis & 537 & $5 \%$ & 586 & $4 \%$ & 1.040 & $5 \%$ & 0,366 \\
\hline Enterococcus faecalis & 430 & $4 \%$ & 440 & $3 \%$ & 832 & $4 \%$ & 0,322 \\
\hline Enterobacter cloacae & 322 & $3 \%$ & 440 & $3 \%$ & 624 & $3 \%$ & 0,982 \\
\hline Acinetobacter baumannii & 322 & $3 \%$ & 293 & $2 \%$ & 208 & $1 \%$ & $\leq 0,001$ \\
\hline Candida albicans & 322 & $3 \%$ & 293 & $2 \%$ & 416 & $2 \%$ & $\leq 0,001$ \\
\hline Proteus mirabilis & 215 & $2 \%$ & 440 & $3 \%$ & 624 & $3 \%$ & $\leq 0,001$ \\
\hline Otros microorganismos & 3.309 & $28 \%$ & 3.810 & $26 \%$ & 6.030 & $29 \%$ & $\leq 0,001$ \\
\hline Total & 10.748 & $100 \%$ & 14.655 & $100 \%$ & 20.792 & $100 \%$ & \\
\hline
\end{tabular}

Cuadro 2. Microorganismos más frecuentes en unidades de cuidados intensivos de 14 hospitales de nivel III de Colombia, años 2006, 2007 y 2008.

\begin{tabular}{|c|c|c|c|c|c|c|c|}
\hline \multirow[t]{3}{*}{ Especie } & \multicolumn{7}{|c|}{ Proporción de aislamientos } \\
\hline & \multicolumn{2}{|c|}{2006} & \multicolumn{2}{|c|}{2007} & \multicolumn{2}{|c|}{2008} & \multirow[t]{2}{*}{$P$} \\
\hline & $\mathrm{n}$ & $\%$ & $\mathbf{n}$ & $\%$ & $\mathbf{n}$ & $\%$ & \\
\hline Escherichia coli & 483 & $9 \%$ & 949 & $12 \%$ & 1.335 & $15 \%$ & $\leq 0,001$ \\
\hline Klebsiella pneumoniae & 473 & $9 \%$ & 949 & $12 \%$ & 979 & $11 \%$ & $\leq 0,001$ \\
\hline Staphylococcus epidermidis & 447 & $8 \%$ & 475 & $6 \%$ & 534 & $6 \%$ & $\leq 0,001$ \\
\hline Staphylococcus aureus & 394 & $7 \%$ & 870 & $11 \%$ & 890 & $10 \%$ & $\leq 0,001$ \\
\hline Acinetobacter baumannii & 381 & $7 \%$ & 475 & $6 \%$ & 267 & $3 \%$ & $\leq 0,001$ \\
\hline Pseudomonas aeruginosa & 344 & $6 \%$ & 712 & $9 \%$ & 801 & $9 \%$ & $\leq 0,001$ \\
\hline Candida albicans & 267 & $5 \%$ & 396 & $5 \%$ & 356 & $4 \%$ & 0,009 \\
\hline Enterobacter cloacae & 170 & $3 \%$ & 237 & $3 \%$ & 267 & $3 \%$ & 0,818 \\
\hline Enterococcus faecalis & 107 & $2 \%$ & 158 & $2 \%$ & 267 & $3 \%$ & $\leq 0,001$ \\
\hline Ptoteus mirabilis & 31 & $1 \%$ & 79 & $1 \%$ & 178 & $2 \%$ & $\leq 0,001$ \\
\hline Otros microorganismos & 2.434 & $44 \%$ & 2.611 & $33 \%$ & 3.026 & $34 \%$ & $\leq 0,001$ \\
\hline Total & 5.531 & $100 \%$ & 7.912 & $100 \%$ & 8.901 & $100 \%$ & \\
\hline
\end{tabular}

generales, se encontró una tendencia significativa al aumento en Enterobacteriaceae (E. coli, P. mirabilisy K. pneumoniae) y $P$. aeruginosa, mientras que $A$. baumannii también mostró tendencia hacia la disminución ( $p \leq 0,001)$ (cuadro 2).

Resistencia de Enterobacteriaceae (E. coli, $K$. pneumoniae y E. cloacae) a cefalosporinas de tercera y cuarta generación en salas generales $y$ unidades de cuidados intensivos

$K$. pneumoniae y E. cloacae se comportaron de manera similar en salas generales, frente a las cefalosporinas de tercera generación. En ambos gérmenes se encontró una disminución estadísticamente significativa de la resistencia para estos antibióticos (figuras 1A y 1B). Por el contrario, la resistencia de $E$. coli frente a los mismos antibióticos fue estable durante los tres años de estudio (figura 1C). El comportamiento de la resistencia a cefepime en estas bacterias no tuvo variaciones importantes durante el período de estudio, excepto para K. pneumoniae que bajó de $10 \%$ a $8 \%(p \leq 0,001)$ (datos sin publicar).

Enlasunidades de cuidados intensivos, nuevamente $K$. pneumoniae presentó disminución significativa en la resistencia a ceftazidime $(p \leq 0,001)$, mientras que frente a ceftriaxona y cefepime se mantuvo sin variaciones (figura 1D, cuadro 3 ). Por el contrario, E. cloacae mostró aumento estadísticamente significante en la resistencia a cefepime (cuadro 3) y no tuvo variaciones significativas frente a ceftazidime o ceftriaxona (figura 1E).

Por otro lado, E. coli tuvo una disminución estadísticamente significante frente a ceftriaxona $(8 \%$ a $7 \%$; $p \leq 0,001)$ y ceftazidime $(6 \%$ a $2 \% ; p \leq 0,001)$ y, al igual que $K$. pneumoniae, no tuvo variaciones importantes frente a cefepime (figura $1 \mathrm{~F}$, cuadro 3). 
A

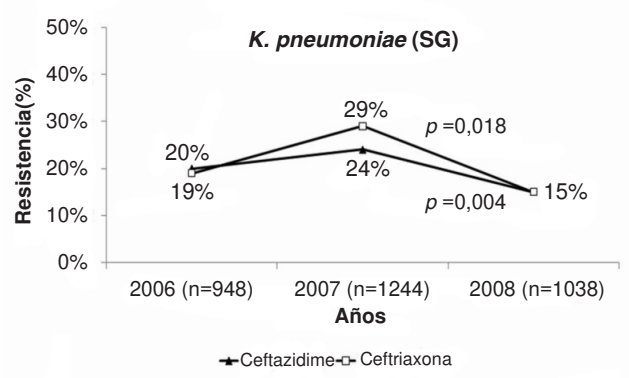

B

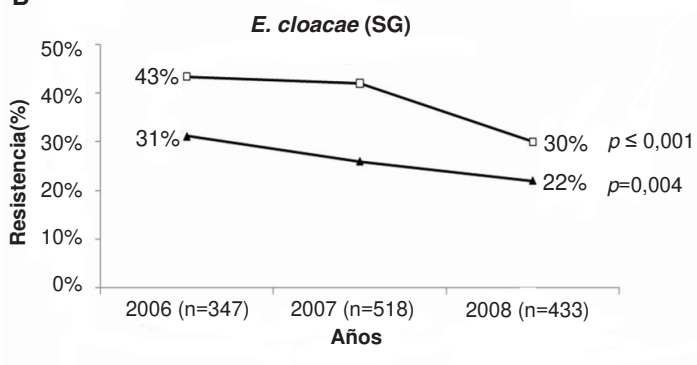

- Ceftazidime- Ceftriaxona

C

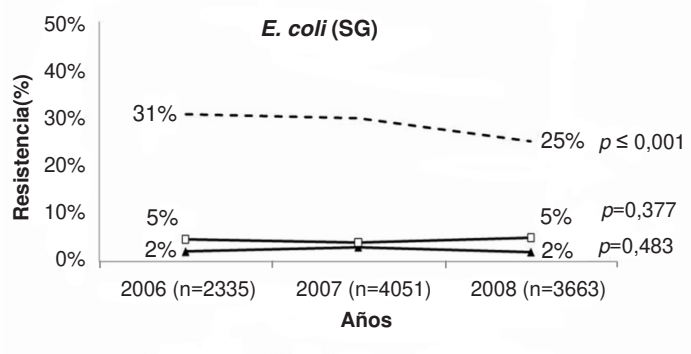

$\rightarrow$ Ceftazidime $-\_$- Ceftriaxona - - Ciprofloxacina
D

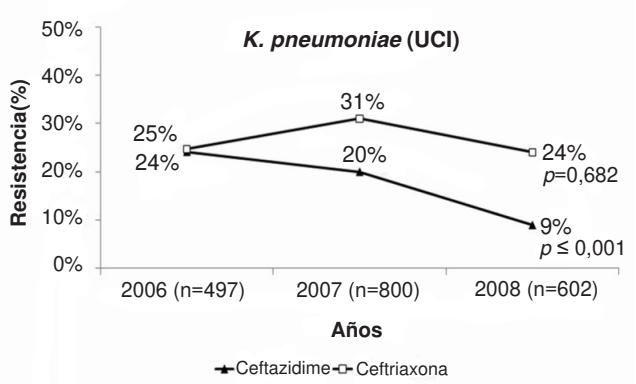

E

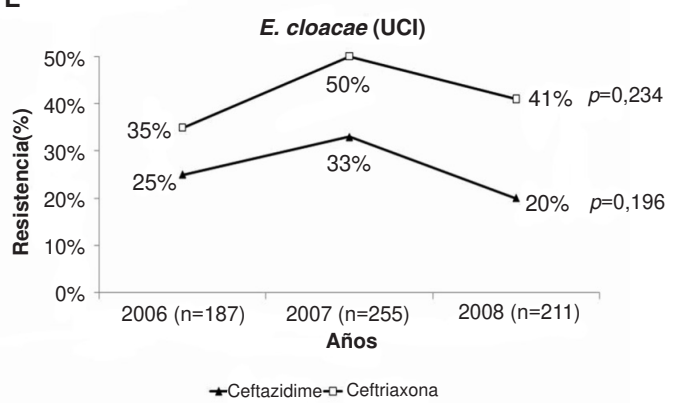

$\mathbf{F}$

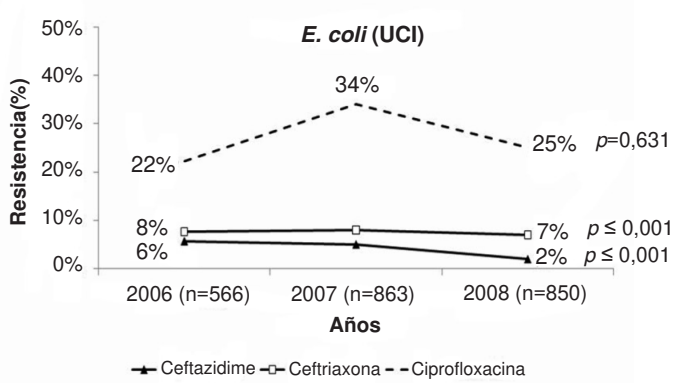

Figura 1. Tendencia en la resistencia de Enterobacteriaceae a antibióticos marcadores en salas generales y unidades de cuidados intensivos de 14 hospitales de nivel III de Colombia, durante los años 2006, 2007 y 2008

Resistencia de Enterobacteriaceae (E. coli, K. pneumoniae y E. cloacae) a piperacilina/ tazobactam, amicacina y ciprofloxacina en salas generales y unidades de cuidados intensivos

En salas generales, ninguno de los tres gérmenes presentó cambios significativos en la resistencia a piperacilina/tazobactam (datos sin publicar). $E$. coli, a pesar de tener la mayor resistencia frente a ciprofloxacina, tuvo una disminución significativa de $31 \%$ a $25 \%$ ( $p \leq 0,001)$ (figura $1 \mathrm{C}$ ).

En las unidades de cuidados intensivos, el comportamiento de los tres gérmenes frente a piperacilina/tazobactam fue estable, sin diferencias significativas para ninguno de ellos (cuadro $3)$. Similar fue lo encontrado en $E$. coli frente a ciprofloxacina (figura 1F).
K. pneumoniae y $E$. coli fueron las únicas Enterobacteriaceae que tuvieron una disminución significativa de la resistencia frente a amicacina (cuadro 3).

Resistencia de Enterobacteriaceae (E. coli, K. pneumoniae y E. cloacae) a carbapenems (imipenem y meropenem) en salas generales $y$ unidades de cuidados intensivos

En general, para los tres gérmenes, en salas generales no hubo cambios significativos en la resistencia a meropenem ni a imipenem (datos sin publicar).

Por otro lado, en las unidades de cuidados intensivos, E. coli se mantuvo $100 \%$ sensible frente a ambos carbapenems, mientras que en $E$. 
Cuadro 3. Resistencia de Enterobacteriaceae a antimicrobianos en unidades de cuidados intensivos de 14 hospitales de nivel III de Colombia, años 2006, 2007 y 2008.

\begin{tabular}{|c|c|c|c|c|}
\hline \multirow[t]{2}{*}{ Especie } & \multicolumn{3}{|c|}{ Resistencia (\%) } & \multirow[b]{2}{*}{$\mathbf{p}$} \\
\hline & 2006 & 2007 & 2008 & \\
\hline \multicolumn{5}{|c|}{ Escherichia coli $\left(\mathrm{n}_{1}=566, \mathrm{n}_{2}=863, \mathrm{n}_{3}=850\right)$} \\
\hline Cefepime & $4 \%$ & $5 \%$ & $5 \%$ & 0,231 \\
\hline Piperacilina/tazobactam & $7 \%$ & $7 \%$ & $6 \%$ & 0,352 \\
\hline Aztreonam & $11 \%$ & $8 \%$ & $8 \%$ & 0,075 \\
\hline Amicacina & $4 \%$ & $3 \%$ & $2 \%$ & 0,2 \\
\hline Meropenem & $0 \%$ & $0 \%$ & $0 \%$ & \\
\hline Imipenem & $0 \%$ & $0 \%$ & $0 \%$ & \\
\hline \multicolumn{5}{|c|}{ Klebsiella pneumoniae $\left(n_{1}=497, n_{2}=800, n_{3}=602\right)$} \\
\hline Cefepime & $12 \%$ & $18 \%$ & $11 \%$ & 0,713 \\
\hline Piperacilina/tazobactam & $20 \%$ & $19 \%$ & $18 \%$ & 0,278 \\
\hline Aztreonam & $37 \%$ & $31 \%$ & $22 \%$ & $\leq 0,001$ \\
\hline Amicacina & $11 \%$ & $5 \%$ & $3 \%$ & 0,002 \\
\hline Meropenem & $1 \%$ & $1 \%$ & $2 \%$ & 0,52 \\
\hline Imipenem & $1 \%$ & $1 \%$ & $1 \%$ & 0,92 \\
\hline \multicolumn{5}{|c|}{ Enterobacter cloacae $\left(n_{1}=187, n_{2}=255, n_{3}=211\right)$} \\
\hline Cefepime & $14 \%$ & $25 \%$ & $23 \%$ & 0,026 \\
\hline Piperacilina/tazobactam & $25 \%$ & $25 \%$ & $22 \%$ & 0,509 \\
\hline Aztreonam & $31 \%$ & $46 \%$ & $32 \%$ & 0,795 \\
\hline Amicacina & $19 \%$ & $17 \%$ & $15 \%$ & 0,28 \\
\hline Meropenem & $3 \%$ & $1 \%$ & $5 \%$ & 0,324 \\
\hline Imipenem & $5 \%$ & $0 \%$ & $1 \%$ & 0,01 \\
\hline
\end{tabular}

$\mathrm{n}_{1}$ : total de aislamientos 2006; $\mathrm{n}_{2}$ : total de aislamientos 2007, $\mathrm{n}_{3}:$ total de aislamientos 2008

cloacae se encontró una disminución significativa en la resistencia a imipenem $(p=0,004)$. En $K$. pneumoniae la resistencia a ambos antibióticos se mantuvo sin variaciones importantes, entre $1 \%$ y $2 \%$ (cuadro 3).

Resistencia de $P$. aeruginosa a piperacilina/ tazobactam y cefepime en salas generales $y$ unidades de cuidados intensivos

En salas generales se observó aumento en la resistencia a ambos antibióticos, pero fue sólo significativo para cefepime (11\% a $15 \%, p=0,011)$ (figura 2A).

Al igual que lo observado en salas generales, la resistencia de $P$. aeruginosa en las unidades de cuidados intensivos frente a cefepime tuvo un incremento de $15 \%$ a $20 \% \quad(p=0,053)$, mientras que el comportamiento de la resistencia frente a piperacilina/tazobactam fue estable en los tres años y estuvo alrededor de 15\% (figura 2B).

Resistencia de gérmenes no fermentadores (A. baumannii y $P$. aeruginosa) a carbapenems (imipenem y meropenem) en salas generales $y$ unidades de cuidados intensivos

El comportamiento de la resistencia a los carbapenems en estos dos gérmenes es disímil.
En salas generales, $P$. aeruginosa, aumentó significativamente su resistencia tanto a imipenem como para meropenem, alcanzando resistencias hasta de $16 \% \quad(p \leq 0,001)$ y $23 \% \quad(p \leq 0,001)$, respectivamente. Sin embargo, estos valores son inferiores a los observados en las unidades de cuidados intensivos, como se mostrará a continuación (figura 2C).

En las unidades de cuidados intensivos, la resistencia mostrada por $P$. aeruginosa no es distinta a la de salas generales; se presentó aumento significativo de la resistencia tanto a imipenem como a meropenem, pasando de $14 \%$ a $28 \%(p \leq 0,001)$ y $16 \%$ a $26 \%(p \leq 0,001)$, respectivamente (figura 2D).

En el caso de $A$. baumannii, se observaron cambios no significativos en salas generales (figura $2 \mathrm{E}$ ), mientras que en las unidades de cuidados intensivos se observó disminución en la resistencia a ambos carbapenems, y fue significativa sólo para meropenem $(p=0,005)$ (figura $2 \mathrm{~F})$.

\section{Discusión}

Los sistemas de vigilancia antimicrobiana tienen como uno de sus principales objetivos aportar conocimiento para disminuir las tasas de 

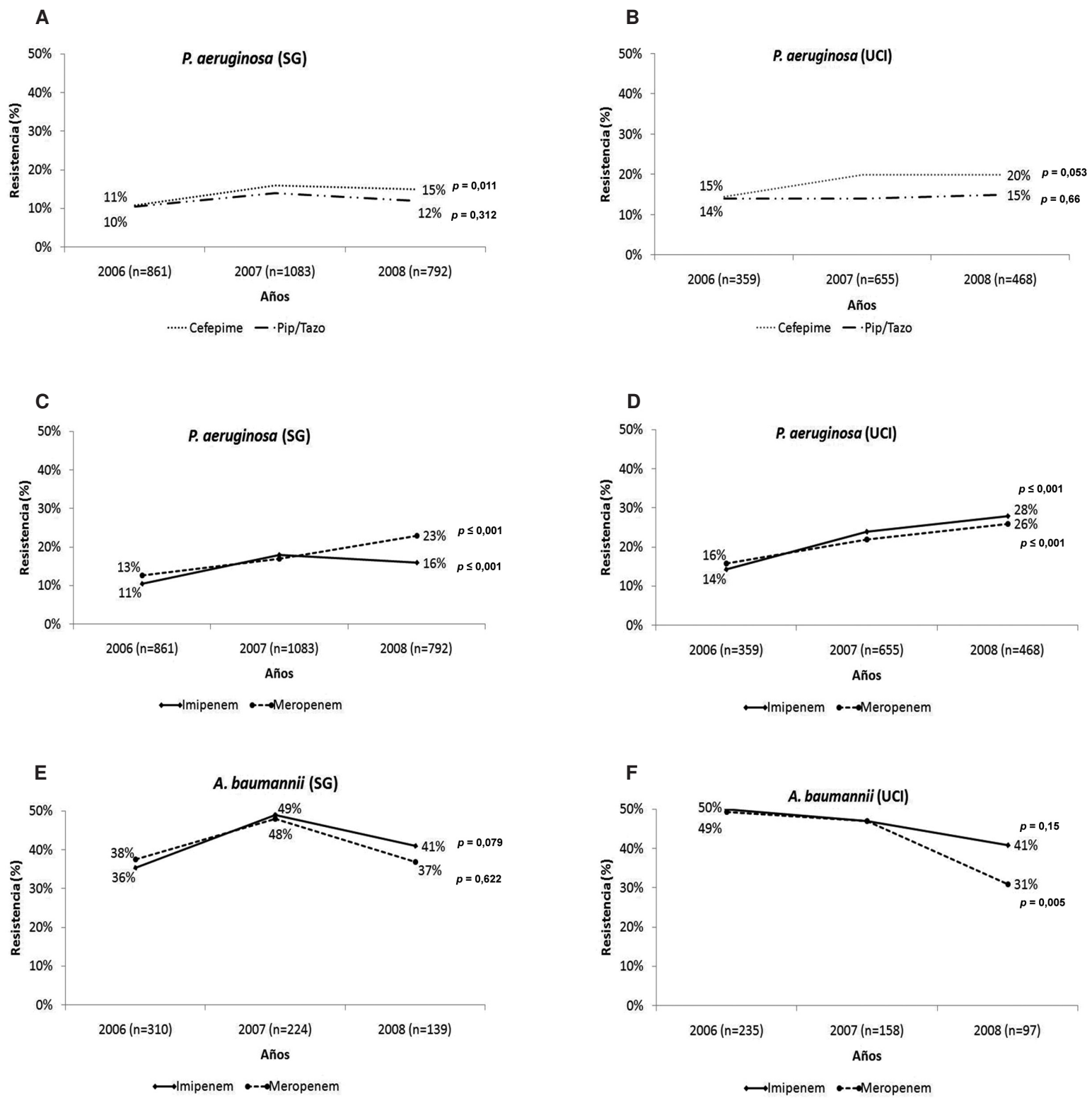

Figura 2. Tendencia en la resistencia de Pseudomonas aeruginosa y Acinetobacter baumannii en salas generales y unidades de cuidados intensivos de 14 hospitales de nivel III de Colombia, durante los años 2006, 2007 y 2008

gérmenes multirresistentes e infecciones hospitalarias (3).

En este estudio se reporta el comportamiento de las frecuencias y el porcentaje de la resistencia antimicrobiana en bacilos Gram negativos durante tres años, periodo en el que se observó un incremento en el número de aislamientos informados cada año tanto en salas generales como en unidades de cuidados intensivos. La distribución de los microorganismos reportados es comparable a lo publicado por SENTRY y MYSTIC, en los cuales los Gram negativos más frecuentes en las unidades de cuidados intensivos durante 19972000 fueron $P$. aeruginosa (22,5\%), E. coli $(19,8 \%)$, Klebsiella spp. (13,9\%), Enterobacterspp. (11,2\%), A. baumannii (6,8\%), Serratia marcescens $(5,1 \%)$ y $P$. mirabilis (5\%) (16). Otros reportes ubican a $E$. coli en primer lugar, con una frecuencia de $19,7 \%(17)$.

La detección de las BLEE se ha considerado como un buen marcador de fenotipo de multirresistencia (18), ya que en las bacterias que la expresan 
se ha observado resistencia simultánea a otros antibióticos, como flouroquinolonas, aminoglucósidos, piperacilina/tazobactam y trimetoprim/ sulfametoxazol $(19,20)$.

Muchos estudios han reportado la restricción del uso de cefalosporinas de tercera generación como una estrategia exitosa en la disminución de la prevalencia de BLEE, ya que ello resultó en una disminución de la resistencia de $K$. pneumoniae a estos antibióticos $(21,22)$. La introducción del lavado de manos y la implementación de barreras de contacto y desinfección, sin la restricción de antibióticos, también ha contribuido a la reducción de las BLEE en el ámbito hospitalario, pero el impacto ha sido de menor magnitud que con la restricción de cefalosporinas de tercera generación (23).

Para E. coli, tanto en salas generales como en unidades de cuidados intensivos, la resistencia a cefalosporinas de tercera generación se ha mantenido en menos de $10 \%$, con una tendencia hacia la disminución para ceftazidime y ceftriaxona. Por otro lado, la resistencia de E. coli a fluoroquinolonas, representada en este informe con ciprofloxacina, es la más alta de todos los antibióticos tamizados.

La resistencia de K. pneumoniae a cefalosporinas de tercera generación, tanto en salas generales como en unidades de cuidados intensivos, sugiere alta prevalencia de BLEE y alcanza frecuencias de resistencia hasta de $31 \%$. Sin embargo, se observó disminución en la resistencia a cefotaxime, ceftriaxona y ceftazidime durante el periodo estudiado.

El uso de cefalosporinas de tercera generación también se ha asociado con la activación de un gen capaz de llevar al aumento en la producción de AmpC por parte de E. cloacae, lo que contribuye a generar falla del tratamiento (24); además de hidrolizar las cefalosporinas de tercera generación, esta $\beta$-lactamasa $(\mathrm{AmpC})$ también puede hidrolizar los monobactámicos y los inhibidores $\beta$-lactámicos (19).

En salas generales, se observó una disminución significativa en la resistencia de E. cloacae a las cefalosporinas de tercera generación y a piperacilina/tazobactam, lo cual podría estar asociado a la restricción del uso de cefalosporinas de tercera generación, ya que evitaría la activación del gen y la hidrólisis de ambos antibióticos. Al igual que para $E$. coli y $K$. pneumoniae productores de BLEE, los carbapenems son la primera opción terapéutica para infecciones graves causadas por bacterias productoras de AmpC (25). La resistencia de Enterobacteriaceae a carbapenems es muy inusual, menor de $5 \%$ en nuestro estudio, y se reporta ya que es importante en el tratamiento de infecciones producidas por bacterias multirresistentes y por ser un marcador para la aparición de carbapenemasas, especialmente de la KPC (Klebsiella pneumoniae carbapenemase) en nuestro país $(10,26)$.

Tanto en salas generales como en unidades de cuidados intensivos, lo encontrado en E. coli y $K$. pneumoniae para carbapenems es similar a lo publicado por SENTRY, en el cual las sensibilidades a estos antibióticos son de $98 \%$ o mayores. Esto es importante ya que, como se mencionó anteriormente, los carbapenems son los antibióticos más eficaces para el tratamiento de las infecciones causadas por Enterobacteriaceae productoras de BLEE $(27,28)$.

En el caso de $P$. aeruginosa, este microorganismo se mantiene como el patógeno multirresistente de mayor prevalencia. Su comportamiento es similar a lo reportado por otros autores, como Oudhuis et al. quienes demostraron, en un periodo de siete años, un aumento significativo en la resistencia a $\beta$-lactámicos, $\beta$-lactámicos/inhibidor$\beta$-lactámico, cefalosporinas de tercera generación, aminoglucósidos y fluoroquinolonas (29).

En los hospitales de la red, se observó que este germen continúa presentando perfiles altos de resistencia tanto en salas generales como en unidades de cuidado intensivo. Esto podría tener un impacto clínico sobre la terapia efectiva contra $P$. aeruginosa multirresistente, ya que obligaría a utilizar, al menos, dos antibióticos de amplio espectro $y$, si la tendencia actual continúa, las opciones terapéuticas futuras serán limitadas (30).

El manejo de $P$. aeruginosa multirresistente requiere de estrategias interdisciplinarias, como un uso racional de antibióticos selectivos contra este germen, reducción del espectro contra Pseudomonas spp. en caso de comprobarse otra bacteria de tipo Enterobacteriaceae (para disminuir la presión selectiva), el uso de conceptos farmacológicos para lograr que el antibiótico alcance concentraciones por encima de la CIM y la vigilancia de brotes que pueden amplificar la resistencia en los hospitales, entre otras; todos estos conceptos ampliamente discutidos hacen que la contención de la resistencia de $P$. aeruginosa sea el mayor reto hospitalario actual y el más difícil (31). 
En $A$. baumannii, a pesar de los altos niveles de resistencia a carbapenems (30\% a $40 \%)$, se observó una tendencia hacia la disminución.

En resumen, la disminución en la proporción de resistencias de algunos gérmenes, se encuentra posiblemente relacionada con la adecuada implementación de protocolos y estrategias de control de infecciones, teniendo en cuenta el reporte de brotes que se realiza por EFCP, así como el manejo racional de antibióticos, según la epidemiología local, y la presencia de $\beta$-lactamasas (especialmente carbapenemasas) que se reporta semestralmente en los informes realizados por el CIDEIM.

Es claro que los programas de vigilancia son necesarios para identificar oportunamente los cambios en el perfil de resistencia de los patógenos causantes de infecciones graves y para supervisar las tendencias en los patrones de sensibilidad que ayudarían a conocer la epidemiologia bacteriana local y a crear protocolos de tratamiento empírico adecuado $(32,33)$.

Aunque los hallazgos presentados en este informe reflejan el consolidado de los hospitales de la red, un estudio individual de cada hospital podría determinar el impacto que las medidas han tenido sobre los perfiles de resistencia reportados en cada uno de ellos.

Es importante mencionar que uno de los factores limitantes del presente estudio es el número pequeño de hospitales con que cuenta la red; sin embargo, se debe seguir trabajando para continuar ampliando la cobertura con el fin de contener la aparición de gérmenes multirresistentes $y$, en general, disminuir las tasas de resistencia bacteriana en el país.

Otra de las limitaciones de este estudio es la ausenciadevariables explicativas predeterminadas, como días de hospitalización, mortalidad, tipo y gravedad de la infección, dosis diaria definida de antibióticos y costos hospitalarios asociados a la infección, lo cual no permite modelar las tendencias de la resistencia bacteriana mediante estudios de series de tiempo, para establecer asociaciones, tendencias y predicciones del comportamiento de este fenómeno en tiempo y espacio definidos.

\section{Agradecimientos}

A Martha Vallejo y a Fernando Rosso por la revisión y comentarios al manuscrito. A Nancy Gore Saravia por su dedicación y aporte para la realización del manuscrito.

\section{Conflicto de intereses}

María Virginia Villegas ha recibido soporte económico para investigación y ha sido asesora de Baxter, Merck Sharp \& Dohme, Pfizer, Wyeth Pharmaceuticals, Bristol-Myers Squibb, AstraZeneca y Janssen.

Los otros autores no reportan conflicto de intereses.

\section{Financiación}

La conformación de la red de hospitales pertenecientes al Grupo para el Estudio de la Resistencia Nosocomial en Colombia se hizo gracias al soporte financiero de: AstraZeneca, Colombia; Pfizer Inc., Groton, CT; Merck Sharp \& Dohme; Baxter, Colombia; Wyeth, Colombia; Janssen, Colombia; y Bristol-Myers Squibb.

\section{Referencias}

1. World Health Organization. WHO Global strategy for containment of antimicrobial resistance. WHO/CDS/CSR/ DRS/2001.2.Geneva: WHO; 2001.

2. Critchley IA, Karlowsky JA. Optimal use of antibiotic resistance surveillance systems. Clin Microbiol Infect. 2004;10:502-11.

3. Tokars JI, Richards C, Andrus M, Klevens M, Curtis A, Horan $\mathrm{T}$, et al. The changing face of surveillance for health care-associated infections. Clin Infect Dis. 2004;39:134752.

4. Jones RN, Mendes C, Turner PJ, Masterton R. An overview of the Meropenem Yearly Susceptibility Test Information Collection (MYSTIC) Program: 1997-2004. Diagn Microbiol Infect Dis. 2005;53:247-56.

5. Falagas ME, Karageorgopoulos DE. Pandrug resistance (PDR), extensive drug resistance (XDR), and multidrug resistance (MDR) among Gram-negative bacilli: need for international harmonization in terminology. Clin Infect Dis. 2008;46:1121-2.

6. Masterton R. The importance and future of antimicrobial surveillancestudies. Clin Infect Dis. 2008;47(Suppl.1):S2131.

7. Kollef MH, Sherman G, Ward S, Fraser VJ. Inadequate antimicrobial treatment of infections: a risk factor for hospital mortality among critically ill patients. Chest. 1999;115:462-74.

8. Leal AL, Eslava-Schmalbach J, Álvarez C, Buitrago G, Méndez M. Endemic tendencies and bacterial resistance markers in third-level hospitals in Bogotá, Colombia. Rev Salud Pública (Bogotá). 2006;8(Suppl.1):59-70.

9. Villegas MV, Correa A, Pérez F, Miranda MC, Zuluaga T, Quinn JP, et al. Prevalence and characterization of extended-spectrum $\beta$-lactamases in Klebsiella pneumoniae and Escherichia coli isolates from Colombian hospitals. Diagn Microbiol Infect Dis. 2004;49:217-22.

10. Villegas MV, Lolans K, Correa A, Suárez CJ, López JA, Vallejo $\mathbf{M}$, et al. First detection of the plasmid-mediated 
class A carbapenemase KPC-2 in clinical isolates of Klebsiella pneumoniae from South America. Antimicrob Agents Chemother. 2006;50:2880-2.

11. Villegas MV, Kattan JN, Correa A, Lolans K, Guzmán AM, Woodford N, et al. Dissemination of Acinetobacter baumannii clones with OXA-23 Carbapenemase in Colombian hospitals. Antimicrob Agents Chemother. 2007;5:2001-4.

12. Miranda MC, Pérez F, Zuluaga T, Olivera MR, Correa A, Reyes SL, et al. Resistencia a antimicrobianos en bacilos Gram negativos aislados en unidades de cuidado intensivo en hospitales de Colombia, WHONET 2003, 2004 y 2005. Biomédica. 2006;26:424-33.

13. Horan TC, Andrus M, Dudeck MA. CDC/NHSN surveillance definition of health care-associated infection and criteria for specific types of infections in the acute care setting. Am J Infect Control. 2008;36:309-32.

14. Clinical and Laboratory Standards Institute. Performance Standards for Antimicrobial Susceptibility Testing; 19th Informational Supplement. CLSI document M100-S19. Wayne, PA: Clinical and Laboratory Standards Institute; 2009.

15. Stelling JM, O'Brien TF. Surveillance of antimicrobial resistance: the WHONET program. Clin Infect Dis. 1997;24(Suppl.1):S157-68.

16. Jones RN. Global epidemiology of antimicrobial resistance among community-acquired and nosocomial pathogens: a five-year summary from the SENTRY Antimicrobial Surveillance Program (1997-2001). Semin Respir Crit Care Med. 2003;24:121-34

17. Quinn JP. Pseudomonas aeruginosa infections in the intensive care unit. Semin Respir Crit Care Med. 2003;24:61-8.

18. Giske CG, Monnet DL, Cars O, Carmeli Y. Clinical and economic impact of common multidrug-resistant Gramnegative bacilli. Antimicrob Agents Chemother. 2008;52: 813-21.

19. Livermore DM, Woodford $\mathbf{N}$. The $\beta$-lactamase threat in Enterobacteriaceae, Pseudomonas and Acinetobacter. Trends Microbiol. 2006;14:413-20.

20. Schwaber MJ, Navon-Venezia S, Schwartz D, Carmeli Y. High levels of antimicrobial coresistance among extended spectrum- $\beta$-lactamase-producing Enterobacteriaceae. Antimicrob Agents Chemother. 2005;49:2137-9.

21. Rahal JJ, Urban C, Horn D, Freeman K, Segal-Maurer $\mathrm{S}$, Maurer $\mathrm{J}$, et al. Class restriction of cephalosporin use to control total cephalosporin resistance in nosocomial Klebsiella. JAMA. 1998;280:1233-7.
22. Rice LB, Eckstein EC, DeVente J, Shlaes DM. Ceftazidimeresistant Klebsiella pneumoniae isolates recovered at the Cleveland Department of Veterans Affairs Medical Center. Clin Infect Dis. 1996;23:118-24.

23. Lucet JC, Decre D, Fichelle A, Joly-Guillou ML, Pernet M, Deblangy C, et al. Control of a prolonged outbreak of extended-spectrum $\beta$-lactamase-producing Enterobacteriaceae in a university hospital. Clin Infect Dis. 1999;29:1411-8.

24. Chow J, Fine M, Shlaes D, Quinn JP, Hooper DC, Johnson MP, et al. Enterobacter bacteremia: Clinical features and emergence of antibiotic resistance during therapy. Ann Intern Med. 1991;115:585-90.

25. Jacoby GA. AmpC beta-lactamases. Clin Microbiol Rev. 2009;22:161-82.

26. Villegas MV, Lolans K, Correa A, Kattan JN, López JA, Quinn JP. First Identification of Pseudomonas aeruginosa isolates producing a KPC-type carbapenemhydrolyzing $\beta$-lactamase. Antimicrob Agents Chemother. 2007;5:1553-5.

27. Paterson DL, Ko WC, Von GA, Mohapatra S, Casellas $\mathbf{J M}$, Goossens $\mathbf{H}$, et al. Antibiotic therapy for Klebsiella pneumoniae bacteremia: implications of production of extended-spectrum $\beta$-lactamases. Clin Infect Dis. 2004;39:31-7.

28. Paterson DL, Depestel DD. Doripenem. Clin Infect Dis. 2009;49:291-8.

29. Oudhuis GJ, Verbon A, Hoogkamp-Korstanje JA, Stobberingh EE. Antimicrobial resistance in Escherichia coli and Pseudomonas aeruginosa from intensive care units in The Netherlands, 1998-2005. Int J Antimicrob Agents. 2008;31:58-63.

30. Gales AC, Jones RN, Turnidge J, Rennie R, Ramphal R. Characterization of Pseudomonas aeruginosa isolates: occurrence rates, antimicrobial susceptibility patterns, and molecular typing in the global SENTRY Antimicrobial Surveillance Program, 1997-1999. Clin Infect Dis. 2001;32(Suppl.2):S146-55

31. Paterson DL. The role of antimicrobial management programs in optimizing antibiotic prescribing within hospitals. Clin Infect Dis. 2006;42(Suppl.2):S90-5.

32. Ibrahim EH, Ward S, Sherman G, Schaiff R, Fraser VJ, Kollef $\mathbf{M H}$. Experience with a clinical guideline for the treatment of ventilator-associated pneumonia. Crit Care Med. 2001;29:1109-15

33. File TM. The science of selecting antimicrobials for community-acquired pneumonia (CAP). J Manag Care Pharm. 2009;15(Suppl.V):S5-11. 